\title{
Novel NCC mutants and functional analysis in a new cohort of patients with Gitelman syndrome
}

\author{
Bob Glaudemans ${ }^{1,3,4}$, Helger G Yntema ${ }^{2,4}$, Pedro San-Cristobal ${ }^{1}$, Jeroen Schoots ${ }^{2}$, Rolph Pfundt ${ }^{2}$, \\ Erik-J Kamsteeg ${ }^{2}$, René J Bindels ${ }^{1}$, Nine VAM Knoers ${ }^{2}$, Joost G Hoenderop ${ }^{1}$ and Lies H Hoefsloot ${ }^{\star, 2}$
}

\begin{abstract}
Gitelman syndrome (GS) is an autosomal recessive disorder characterized by hypokalemic metabolic alkalosis in conjunction with significant hypomagnesemia and hypocalciuria. The GS phenotype is caused by mutations in the solute carrier family 12 , member 3 (SLC12A3) gene that encodes the thiazide-sensitive NaCl cotransporter (NCC). We analyzed DNA samples of 163 patients with a clinical suspicion of GS by direct sequencing of all 26 exons of the SLC12A3 gene. In total, 114 different mutations were identified, 31 of which have not been reported before. These novel variants include 3 deletions, 18 missense, 6 splice site and 4 nonsense mutations. We selected seven missense mutations to investigate their effect on NCC activity and plasma membrane localization by using the Xenopus laevis oocyte expression system. The Thr392/le mutant did not display transport activity (probably class 2 mutation), while the Asn442Ser and GIn1030Arg NCC mutants showed decreased plasma membrane localization and consequently function, likely due to impaired trafficking (class 3 mutation). Even though the $\mathrm{NaCl}$ uptake was hampered for NCC mutants Glu121Asp, Pro751Leu, Ser475Cys and Tyr489His, the transporters reached the plasma membrane (class 4 mutation), suggesting an effect on NCC regulation or ion affinity. The present study shows the identification of 38 novel mutations in the SLC12A3 gene and provides insight into the mechanisms that regulate NCC. European Journal of Human Genetics (2012) 20, 263-270; doi:10.1038/ejhg.2011.189; published online 19 October 2011
\end{abstract}

Keywords: Gitelman syndrome; SLC12A3; mutation analysis; NCC; Xenopus laevis oocyte expression

\section{INTRODUCTION}

Gitelman syndrome (GS) (OMIM 263800) is an autosomal recessive renal tubular salt wasting disorder, primarily characterized by hypokalemic metabolic alkalosis in combination with significant hypomagnesemia and low urinary calcium. ${ }^{1}$ GS is the most frequently occurring renal tubular disorder with a prevalence of 1:40000 in the Caucasian population (heterozygotes 1:100). ${ }^{2}$ The GS phenotype can be observed from the age of 6 years, but in most instances the diagnosis is made during adulthood. ${ }^{3}$ Transient periods of muscle weakness and tetany ${ }^{1,4}$ sometimes accompanied by abdominal pain, vomiting and fever are often seen in GS patients. Remarkably, some patients are completely asymptomatic except for the appearance of chondrocalcinosis at adult age.,

A minority of patients with GS have a mutation in the CLCNKB gene, coding for chloride channel subunit b $(\mathrm{ClC}-\mathrm{Kb})$. In most cases, GS results from loss-of-function mutations in the solute carrier family 12 , member 3 (SLC12A3) gene, which consists of 26 exons and is located on the long arm of chromosome 16 (16q13). The SLC12A3 gene encodes the 1030 amino-acid thiazide-sensitive $\mathrm{NaCl}$ cotransporter (NCC) protein (NM_000339.2; OMIM 600968). ${ }^{7}$ The two-dimensional structure of NCC is predicted to contain 12 transmembrane segments (S) with large intracellular amino (N)- and carboxy (C)-terminal regions, and a large hydrophilic extracellular loop between S7 and S8. ${ }^{8}$ NCC localizes to the luminal membrane of the DCT segment of the nephron, ${ }^{9}$ where it facilitates the cotransport of $\mathrm{Na}^{+}$and $\mathrm{Cl}^{-}$from the pro-urine to the intracellular compartment.
NCC is the target for thiazide-type diuretics, which are currently recommended as the first-line pharmacological treatment of hypertension. ${ }^{10}$ Chronic thiazide administration, on the other hand, induces similar electrolyte disturbances as seen in GS. ${ }^{11}$

At present, $\sim 250$ distinct loss-of-function mutations have been discovered in patients with GS (Human Gene Mutation Database, http://biobase-international.com). Most are missense mutations substituting a conserved amino-acid residue, whereas nonsense, frameshift and splice site mutations and gene rearrangements are less frequently observed. These mutations presumably impair the function of the encoded NCC protein as a result of structural changes and/or altered cellular localization. So far, functional analysis has been performed for only a limited number of mutations. ${ }^{12-15}$ To confirm disease-causing NCC mutants, Xenopus laevis oocytes were used as a model system to investigate $\mathrm{Na}^{+}$transport by NCC and cell surface localization. These mutants are categorized according to standard nomenclature as established for renal $\mathrm{Na}^{+}$transporter's NCC and NKCC2 mutations. ${ }^{16}$

Here, we report the identification of 31 novel SLC12A3 sequence variants, that are predicted to result in loss of function of the SLC12A3 protein and are considered to be disease-causing mutations. We selected seven missense mutations (five novel mutations and two previously described) to characterize their functional and molecular properties by performing a quantitative analysis comparing wild-type to mutant NCC plasma membrane expression as well as by evaluating their NCC transport activity. The present study expands the spectrum

${ }^{1}$ Department of Physiology, Radboud University Nijmegen Medical Centre, Nijmegen, The Netherlands; ${ }^{2}$ Department of Human Genetics, Radboud University Nijmegen Medical Centre, Nijmegen, The Netherlands; ${ }^{3}$ Institute of Physiology, University of Zurich, Zurich, Switzerland

*Correspondence: Dr LH Hoefsloot, Department of Human Genetics (848), Radboud University Nijmegen Medical Centre, PO Box 9101,6500 HB Nijmegen, The Netherlands. Tel: +31 24361 3799; Fax: +31 24356 5026; E-mail: I.hoefsloot@antrg.umcn.nl

4These authors have contributed equally to this work.

Received 20 June 2011; revised 14 September 2011; accepted 15 September 2011; published online 19 October 2011 
of SLC12A3 mutations and evaluates their effect on NCC function to ultimately elucidate the molecular mechanisms underlying GS.

\section{MATERIALS AND METHODS}

\section{Mutation analysis}

Patient samples were sent to the DNA diagnostic service of the Department of Human Genetics of our hospital for diagnostic analysis of the SLC12A3 gene. Most samples were sent from nephrology, endocrinology and genetic services in the Netherlands, but samples from other European countries (eg, Belgium, Sweden and Italy) were also tested. We relied on the expertize of the referring medical doctors for inclusion of patients for diagnostic testing, and we did not actively ask for clinical details of patients. However, all patients with a clinical description on the request form (about $75 \%$ of cases) fulfilled the classical criteria for GS. DNA was extracted from peripheral blood lymphocytes using standard procedures. All individual exons of the SLC12A3 gene were PCR amplified (primers and PCR conditions available upon request), and direct sequencing was performed on an ABI 3730 automated DNA sequencer (Life Technologies Division Applied Biosystems, Nieuwerkerk a/d IJssel, The Netherlands). The segregation of mutations has been studied in case family members were available. GenBank accession number NM_000339.2 is used as a reference sequence, in which the A of ATG is number 1. Intron sequences can be found in NT_010498.15.

\section{Multiplex ligand-dependent probe amplification}

For the multiplex ligation-dependent probe amplification (MLPA) analysis, the SALSA MLPA kit P136 (MRC-Holland, Amsterdam, The Netherlands) was used. This kit contains 25 MLPA probes covering all exons, except exon 6 of the SLC12A3 gene. Furthermore, a home-designed kit was used containing synthetic probes including all coding exons (also exon 6). The analysis and data processing were performed as described by Koolen et al. ${ }^{17}$

\section{DNA constructs}

To allow hNCC detection at the plasma membrane of X. laevis oocytes, enhanced green fluorescent protein (eGFP) was introduced at the N-terminus of hNCC. The eGFP-hNCC pT7Ts construct was obtained by replacement of the N-terminal flag-tag of the previously described flag-hNCC pT7Ts construct $^{12}$ by the eGFP from the eGFP-hNCC pCB7 construct using Ncol and Nael restriction enzymes (New England Biolabs, Bioke, Leiden, The Netherlands). All selected mutations were introduced into eGFP-hNCC pT7Ts by use of the Quikchange Site-Directed Mutagenesis Kit (Stratagene, Bio-Connect BV, Huissen, The Netherlands), according to the manufacturer's instructions. The primers are depicted in Supplementary Table 2.

\section{In vitro NCC cRNA translation}

To prepare cRNA, all clones were linearized using NsiI (New England Biolabs), and cRNA was in vitro transcribed using a $\mathrm{T}_{7}$ RNA polymerase mMESSAGE machine kit (Ambion, Life Technologies Division Applied Biosystems), according to manufacturer directions. Additionally, all samples were resolved on a $1 \%$ $\mathrm{w} / \mathrm{v}$ formaldehyde agarose gel to evaluate the cRNA quality, while the concentration was determined by absorbance reading at $260 \mathrm{~nm}$ using the NANODROP 2000c (Thermo scientific, Breda, The Netherlands). cRNA aliquots were stored at $-80^{\circ} \mathrm{C}$.

\section{X. laevis oocyte preparation}

The animal ethics board of the Radboud University Nijmegen Medical Centre approved the animal studies. Microinjection, isolation and ${ }^{22} \mathrm{Na}^{+}$tracer uptakes in X. laevis oocytes were performed as described previously, ${ }^{18}$ with minor modifications. In brief, oocytes were harvested by postmortem surgery (abdominal laparotomy) after decapitation of adult female X. laevis and incubated in frog Ringer ND96 $(96 \mathrm{~mm} \mathrm{NaCl}, 2 \mathrm{~mm} \mathrm{KCl}, 1.8 \mathrm{mM} \mathrm{CaCl} 2$, $1 \mathrm{~mm} \mathrm{MgCl}$ and $5 \mathrm{~mm}$ Hepes/Tris, $\mathrm{pH}$ 7.4) in the presence of collagenase B $(2 \mathrm{mg} / \mathrm{ml})$ for $1 \mathrm{~h}$. The oocytes were washed four times in ND96, defolliculated and incubated overnight at $18^{\circ} \mathrm{C}$ in ND96 supplemented with $2.5 \mathrm{~mm}$ sodium pyruvate and $5 \mathrm{mg} / 100 \mathrm{ml}$ of gentamicin. Next day, mature oocytes were microinjected with $50 \mathrm{ml}$ of water only or containing cRNA (10 ng of cRNA/oocyte). The oocytes were incubated at $18^{\circ} \mathrm{C}$ for 3 days in ND96 with
$2.5 \mathrm{~mm}$ sodium pyruvate and $5 \mathrm{mg} / 100 \mathrm{ml}$ gentamicin that was changed every $24 \mathrm{~h}$. The night before the uptake experiments were performed, the oocytes were incubated in $\mathrm{Cl}^{-}$-free ND96 $\left(96 \mathrm{mM} \mathrm{Na}^{+}\right.$-isethionate, $2 \mathrm{~mm} \mathrm{~K}{ }^{+}$-gluconate,

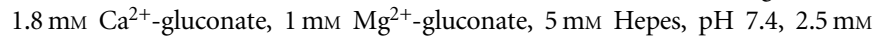
sodium pyruvate and $5 \mathrm{mg} / 100 \mathrm{ml}$ gentamicin).

\section{$X$. laevis oocyte total membrane isolation}

For the isolation of the total membrane fraction, oocytes were homogenized in $20 \mu \mathrm{l} \mathrm{Hba}$ buffer (20 mm TRIS, pH 7.4, $5 \mathrm{~mm} \mathrm{NaH} \mathrm{NO}_{4}, 1 \mathrm{~mm}$ EDTA, $80 \mathrm{~mm}$ sucrose, $1 \mathrm{~mm}$ PMSF, $1 \mu \mathrm{g} / \mathrm{ml}$ leupeptin and $10 \mu \mathrm{g} / \mathrm{ml}$ pepstatin) per oocyte, and centrifugated twice for $10 \mathrm{~min}$ at $100 \mathrm{~g}$ at $4{ }^{\circ} \mathrm{C}$ (each time the supernatant was collected). Following membranes were isolated by $20 \mathrm{~min}$ centrifugation at $14000 \mathrm{~g}$. The pellet was resuspended in $2 \mu \mathrm{l}$ leammli/DTT per oocyte.

\section{Western blotting}

eGFP-NCC expression was analyzed by immunoblot analysis for the total plasma membrane fraction. Protein samples were incubated for $10 \mathrm{~min}$ at room temperature in leammli buffer, subjected to electrophoresis on a $10 \% \mathrm{w} / \mathrm{v}$ SDS-polyacrylamide gel and immunoblotted onto PVDF membranes (Millipore Corporation, Amsterdam, The Netherlands) according to standard procedures. Membranes were blocked for $1 \mathrm{~h}$ in $5 \% \mathrm{w} / \mathrm{v}$ non-fat dried milk in TBS-T (TBS with $0.1 \% \mathrm{v} / \mathrm{v}$ Tween-20). Overnight, blots were incubated with a rabbit polyclonal BFP antibody (1:5000 in $1 \% \mathrm{w} / \mathrm{v}$ milk TBS-T) ${ }^{19}$ following 2-h incubation with a PO-conjugated anti-rabbit antibody (1:10 000 in 1\% w/v milk TBS-T) (Sigma, Zwijndrecht, The Netherlands, no. A 491). The blots were incubated with SuperSignal West Pico (Thermo scientific) before visualization by chemiluminescence in the chemidoc XRS imager (Biorad, Veenendaal, The Netherlands).

\section{${ }^{22} \mathrm{Na}^{+}$transport assays}

Human eGFP-NCC cotransporter function was determined by assessing tracer ${ }^{22} \mathrm{Na}^{+}$uptake (PerkinElmer Life Sciences, Groningen, The Netherlands) in groups of 25 oocytes following a standard protocol of $30 \mathrm{~min}$ of incubation in a $\mathrm{Cl}^{-}$-free ND96 medium containing $1 \mathrm{~mm}$ ouabain, $0.1 \mathrm{~mm}$ amiloride and $0.1 \mathrm{~mm}$ bumetanide, following $1 \mathrm{~h}$ of uptake in $\mathrm{K}^{+}$-free, $\mathrm{NaCl}$ medium ( $40 \mathrm{~mm} \mathrm{NaCl}, 56 \mathrm{~mm}$ sodium-gluconate, $4 \mathrm{~mm} \mathrm{CaCl}_{2}, 1 \mathrm{~mm} \mathrm{MgCl}_{2}$ and $5 \mathrm{~mm}$ Hepes/Tris, pH 7.4) containing $1 \mathrm{~mm}$ ouabain, $0.1 \mathrm{~mm}$ amiloride, $0.1 \mathrm{~mm}$ bumetanide and $2 \mu \mathrm{Ci}$ of ${ }^{22} \mathrm{Na}^{+} / \mathrm{ml}^{20}$ As a control, one group of waterinjected oocytes has been included to determine the basal unspecific tracer ${ }^{22} \mathrm{Na}^{+}$uptake in all experiments.

\section{Immunofluorescence microscopy}

Human eGFP-NCC cotransporter X. laevis oocyte membrane localization was assessed in groups of $\sim 10$ oocytes by use of confocal microscopy. ${ }^{21}$ The images were obtained with an Olympus FV1000 Confocal Laser Scanning Microscope (Olympus, Center Valley, PA, USA), emitting at $488 \mathrm{~nm}$ with a 30-mW argon laser. The plasma membrane fluorescence was quantified for all images by measuring pixel intensity using ImageJ (Image Processing Program, NIH, USA) software.

\section{Statistical analysis}

In all experiments, the data are expressed as mean \pm SEM. Overall statistical significance was determined by one-way ANOVA, followed by a Bonferroni post-hoc test. $P<0.05$ was considered significant.

\section{RESULTS}

Screening for SLC12A3 mutations by sequence analysis

We performed sequence analysis of the entire coding region and flanking splice sites of the SLC12A3 gene, revealing 303 mutations in 163 patients with a clinical suspicion of GS (Supplementary Table 1). Mutations were identified on both SLC12A3 alleles in 138 patients. A total of 39 patients carry homozygous SLC12A3 mutations whereas 99 are compound heterozygotes. If available, segregation analysis of SLC12A3 mutations was performed in family members 
of these probands in order to prove that the mutations are located on separate alleles (data not shown). A total of 25 patients displayed a single mutation in the SLC12A3 gene. (Supplementary Table 1). A large fraction of the identified mutations $(\sim 90 \%)$ has been described previously. $7,12,14,22-26$ The most frequently encountered mutations in our cohort were c.815T $>$ C (p.Leu272Pro; 22/326 alleles), c.2221G $>$ A (p.Gly741Arg; 35/326 alleles) and c.2883+1G $>$ T (27/526 alleles) (Supplementary Table 1). In total, 28 novel mutations were detected by sequencing, including 18 missense, 4 nonsense and 6 splice site mutations (Table 1). An in-silico-based method was used to assess the effect of a mutation on splicing (splice prediction tool of the Alamut software (http://www.interactivebiosoftware.com/alamut/doc/1.5/index. $\mathrm{html})$ ), which was also applied as an intermediate to investigate the effect of the different missense mutations on the protein. Only variants that are classified as UV3 or UV4, according to the guidelines of the CMGS and VKGL (British and Dutch molecular genetic societies), are included as mutations in this paper (see http://www.cmgs.org/BPGs/ pdfs\%20current\%20bpgs/UV\%20GUIDELINES\%20ratified.pdf). These criteria include, among others, the Grantham score, SIFT and PolyPhen analysis, earlier description in the literature, splice site analysis and analysis of frequency of the mutation in the population.

\section{Screening for SLC12A3 deletions by MLPA}

To investigate the occurrence of deletions or duplications, multiplex ligation-dependent probe amplification (MLPA) analysis was performed for cases with a heterozygous mutation, and for cases with a homozygous mutation in which segregation in the family could not be confirmed. In these patients, we identified six heterozygous deletions, which affect at least part of the SLC12A3 coding region. Moreover, in patient 94, whom exons 5 and 6 could not be amplified by PCR analysis, a homozygous deletion of both exons was discovered. All deletions detected in this study are predicted to result in a frameshift and premature translational termination (Table 1; Supplementary Table 1). Taken together, we identified 114 different mutations, 31 of which have not been reported before. These novel variants include 3 deletions, 18 missense, 6 splice site and 4 nonsense mutations.

\section{Wild-type and mutant NCC transport activity}

At present, SLC12A3 missense mutations have been shown to affect $\sim 220$ of the in total 1030 NCC amino acids. Nevertheless, the functional consequence of most mutations has not been investigated. A total of seven identified NCC missense mutations, including five previously unidentified (Glu121Asp, Asn442Ser, Tyr489His, Pro751Leu and Gln1030Arg) and two known variants (Ser475Cys and Thr392Ile) were selected to study their effect on NCC function. Thr392Ile was picked, as it appeared de novo together with the Asn442Ser variant in one patient. These NCC mutations affect amino acids, which are highly conserved among species (Figure 1a). The localization of each examined mutant in the proposed topology of NCC is shown in Figure 1b. The Glu121Asp mutation is located within the N-terminus, which contains important phosphorylation residues (Thr46, Thr55 and Thr60). The Thr392Ile and Asn442Ser mutations are positioned in or in close proximity of the large extracellular loop, the Ser475Cys and Tyr489His are located in the fourth intracellular loop, and the Pro751Leu and Gln1030Arg mutations reside in the C-terminus. The Gln1030Arg mutation affects the last residue of NCC. This residue could be part of a protein-protein interaction motif, which could be important for cell membrane trafficking. The functionality of each mutant NCC transporter was investigated using the $X$. laevis oocyte heterologous expression system. To compare ${ }^{22} \mathrm{Na}^{+}$uptake between wild-type and each mutant NCC,
Table 1 Novel SLC12A3 sequence variants in patients with clinical suspicion of GS

\begin{tabular}{|c|c|c|c|}
\hline $\begin{array}{l}\text { Mutation } \\
\text { type }\end{array}$ & $\begin{array}{l}\text { Nucleotide } \\
\text { change }\end{array}$ & Exon & $\begin{array}{l}\text { Predicted effect on } \\
\text { coding sequence }\end{array}$ \\
\hline \multirow[t]{18}{*}{ Missense } & c. $184 \mathrm{G}>\mathrm{C}$ & 1 & p.Asp62His \\
\hline & c. $363 G>C$ & 2 & p.Glu121Asp \\
\hline & c. $470 \mathrm{~T}>\mathrm{C}$ & 3 & p.Leu157Pro \\
\hline & c. $473 G>T$ & 3 & p.Arg158Leu \\
\hline & c. $575 \mathrm{~T}>\mathrm{C}$ & 4 & p.lle192Thr \\
\hline & c. $581 \mathrm{C}>\mathrm{T}$ & 4 & p.Thr194lle \\
\hline & c. $704 C>G$ & 5 & p.Thr235Arg \\
\hline & c. $775 \mathrm{G}>\mathrm{A}$ & 6 & p.Asp259Asn \\
\hline & c. $1325 A>G$ & 10 & p.Asn442Ser \\
\hline & c. $1465 T>C$ & 12 & p.Tyr489His \\
\hline & c. $1601 A>G$ & 13 & p.Asn534Ser \\
\hline & c. $1679 C>G$ & 14 & p.Pro560Arg \\
\hline & c. $2204 C>G$ & 18 & p.Pro735Arg \\
\hline & c. $2252 \mathrm{C}>\mathrm{T}$ & 18 & p.Pro751Leu \\
\hline & c. $2497 \mathrm{~T}>\mathrm{A}$ & 21 & p.Ser833Thr \\
\hline & c. $2572 \mathrm{C}>\mathrm{T}$ & 22 & p.Leu858Phe \\
\hline & c. $2642 T>C$ & 22 & p.Met881Thr \\
\hline & c. $3089 A>G$ & 26 & p.GIn1030Arg \\
\hline \multirow[t]{4}{*}{ Nonsense } & c. $1024 G>T$ & 8 & p.Gly342X \\
\hline & c. $2164 \mathrm{C}>\mathrm{T}$ & 17 & p.GIn722X \\
\hline & c. $2821 \mathrm{G}>\mathrm{T}$ & 24 & p.Glu941X \\
\hline & c. $2869 \mathrm{~A}>\mathrm{T}$ & 24 & p.Lys957X \\
\hline \multirow[t]{6}{*}{ Splice site } & c. $505+5 G>A$ & Intron 3 & Splice defect \\
\hline & c. $602-16 \mathrm{G}>\mathrm{A}$ & Intron 3 & Splice defect \\
\hline & c. $1096-1 G>A$ & Intron 8 & Splice defect \\
\hline & c. $1335+1 \mathrm{G}>\mathrm{A}$ & Intron 10 & Splice defect \\
\hline & c. $1335+1 G>C$ & Intron 10 & Splice defect \\
\hline & c. $2037+4 A>G$ & Intron 16 & Splice defect \\
\hline \multirow[t]{3}{*}{ Deletion } & c.-66_23del & & Partial deletion exon 1 \\
\hline & $(?--6) \_852+? \mathrm{del}$ & & Deletion exons 1-6 \\
\hline & c.602-?_852+?del & & Deletion exons 5 and 6 \\
\hline
\end{tabular}

Abbreviation: GS, Gitelman syndrome.

GenBank accession number NM 000339.2 is used as a reference sequence, in which the A of ATG is number 1. Intron sequences can be found in NT_010498.15.

all groups of oocytes were injected with $10 \mathrm{ng}$ cRNA/oocyte. At the same day of the NCC-mediated ${ }^{22} \mathrm{Na}^{+}$uptake experiments, in parallel, microinjected $X$. laevis oocytes were selected to determine total membrane NCC protein expression using western blotting analysis. As shown in Figure 2a, total NCC expression was identical in all tested mutants. ${ }^{22} \mathrm{Na}^{+}$uptake by wild-type and mutant NCC-expressing oocytes was performed in the absence and presence of the thiazide diuretic, metalozone. In comparison with wild-type $(100 \% \pm 8 \%)$, the thiazide-sensitive ${ }^{22} \mathrm{Na}^{+}$uptake was significantly diminished for all

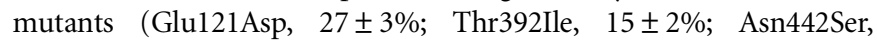
$68 \pm 6 \%$; Ser475Cys, $40 \pm 5 \%$; Tyr 489 His, $48 \pm 5 \%$; Pro751Leu, $54 \pm 4 \%$; and Gln1030Arg, $58 \pm 6 \%$ ) ( $\%$ relative to wild-type, $n=53-69$ oocytes) (Figure $2 \mathrm{~b}$ ). In the presence of thiazides, wildtype and mutant NCC-expressing oocytes showed equal levels of ${ }^{22} \mathrm{Na}^{+}$uptake as $\mathrm{H}_{2} \mathrm{O}$-injected oocytes, demonstrating specific tracer uptakes for wild-type- and mutant-mediated NCC uptake (data no shown). Interestingly, the transport activity of the Thr392Ile mutant appeared to be totally abolished (similar levels of uptake for the Thr392Ile mutant in the absence and presence of thiazides or $\mathrm{H}_{2} \mathrm{O}$-injected oocytes) (data no shown). Decreased transport activity, as observed for each NCC mutant, can result from impaired processing, disrupted membrane internalization or reduced function at the 
Human

Chimpanzee

Rabbit

Rat

Mouse

Cow

Horse

Zebrafish

Winter flounder

\begin{tabular}{|c|c|}
\hline & Ser475Cys \\
\hline Human & $471 \cdot A C L V S$ A A K V $=479$ \\
\hline Chimpanzee & $471 \cdot A C L V S A A K V=479$ \\
\hline Rabbit & $469 \cdot A C L V S A A K V=477$ \\
\hline Rat & $469 \cdot A C L V S$ A A K V $=477$ \\
\hline Mouse & $468 \cdot A C L V S A A K V=476$ \\
\hline Cow & $471 \cdot A C L V S$ A A K V -479 \\
\hline Hors & A A K V -479 \\
\hline Zebrafish & $S$ A P K V -461 \\
\hline Winter flounder & $470 \cdot A C L V S$ A P K V 478 \\
\hline
\end{tabular}

\section{(2) Thr392lle}

388. A I S A T | G S C.396 389. A I S A T | G S C 396 386. A I S A T I G S C.394 386. A | S A T | G S C.395 385. A | S A T | G S C 393 388. A I S A T | G S C 396 388. A I S A T | G S C 396 370. I I S A T I G S C. 378 385. I I S A T I G A C -393

(5) Tyr489His

485. E D Q L Y P L I G . 493 485. E D Q L Y P L | G . 493 483. E D Q L Y P L | G .491 483. E D Q L Y P L | G .491 482. E D Q L Y P L | G 4490 485. L D Q L Y P L | G .493 485. V D Q L Y P L | G .493 $467 \cdot$ K D K L Y P G | G 4.475 484.K D Q L Y P L | G . 492

(3) Asn442Ser

438. Y G L I N Y Y Q T . 446 438. $Y G L \mid N Y Y Q T \cdot 446$ 436. Y G L I N Y Y Q T .444 436. Y G L I N Y Y Q T .444 435. Y G L I N Y Y Q T.443 438. Y G L I N Y Y Q T $\cdot 446$ 438. Y G L I N Y Y Q S .445 420. Y G L S N Y Y O S. 428 437. HG I MN Y Y Q S .445

6 Pro751Leu

747. Q S A HP A TVE .755 747. O S A HP A T VE .755 745. Q S A HP A TVE .753 745. Q S A HP A TVE $\cdot 753$ 744. Q S A HP A T VE .752 747. Q S A HP A T VE .755 747. Q S A HP A TVE 755 727. R K V QP G I I E 735 739. G C D S P Q A A H .747

\section{(7) Gln1030Arg}

1022. E N V L T F Y C Q -1030 1022. EN V L T F Y C $0-1030$ 1022- E N V L T F Y C Q 1030 994- EN V L T F Y C Q -1002 993. EN V L T F Y C - 1001 1022. E N V L T F Y C Q 1030 1022. E N V L T F Y C Q 1030 1004. E N V L T Q Y C Q - 1012 1015- E N V L T F Y C Q -1023

b

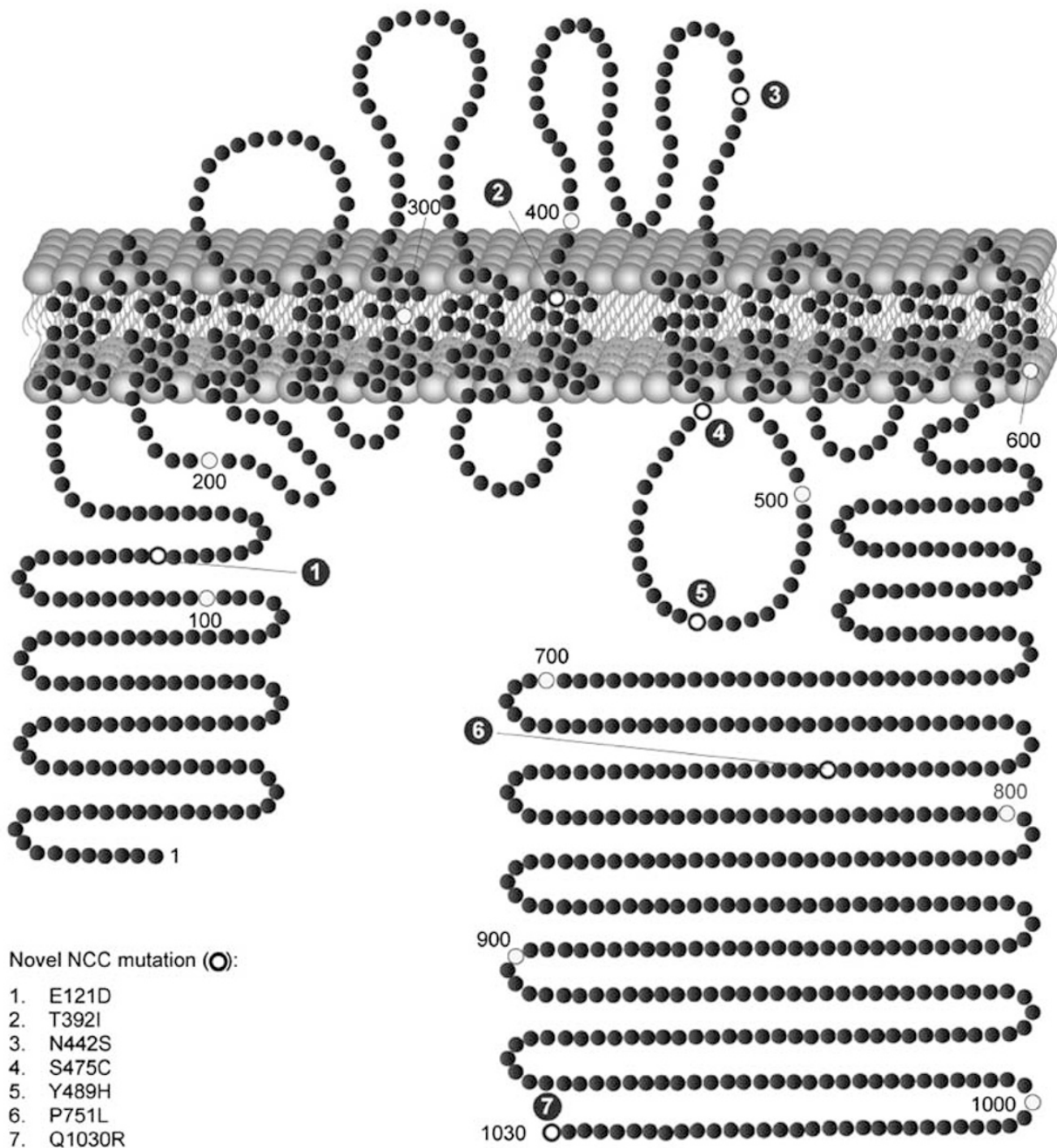

Figure 1 Multiple alignment and predicted topological localization of each NCC mutant. (a) Multiple alignment analysis shows conservation among species of the identified NCC mutant amino acids (gray bar). Gray and black letters represent conserved and non-conserved amino acids, respectively. (b) Schematic topological representation of NCC, which consists of large intracellular N- and C-terminal domains, which are located within the cell, 12 transmembrane segments (S) and a large hydrophilic extracellular loop between S7 and S8 comprising two glycosylation sites. Every dot represents one amino acid and the localization of the functionally characterized NCC mutations is denoted by a white dot (numbers 1-7). 

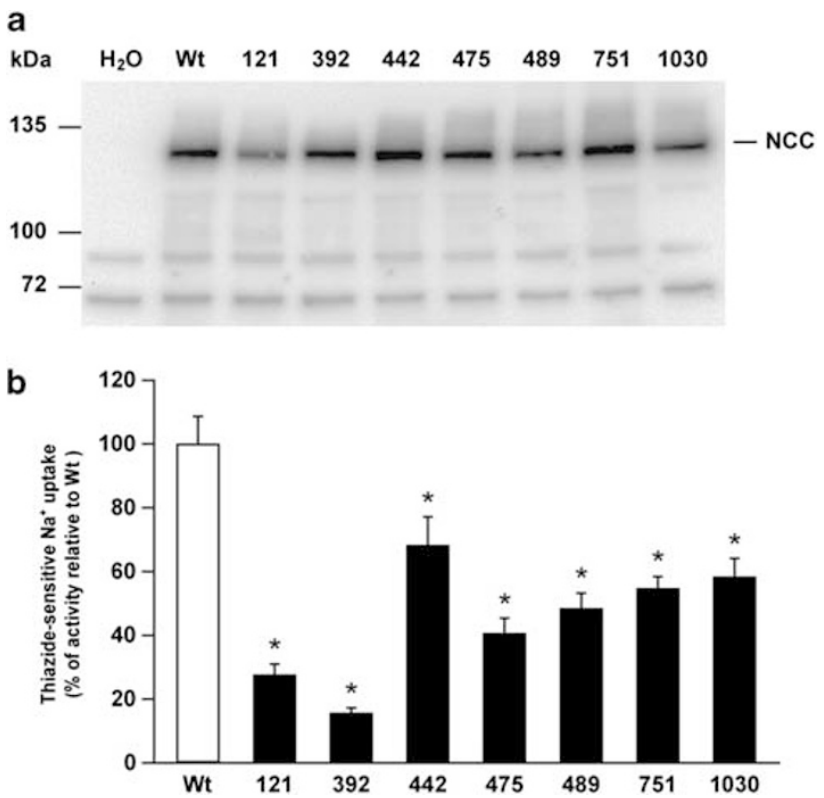

Figure 2 Expression and functional consequence of exogenous wild-type (wt) and mutant NCC in $X$. laevis oocytes. (a) Western blotting analysis of the total membrane fraction of oocytes injected with $\mathrm{H}_{2} \mathrm{O}$, and wt or mutant NCC cRNA. (b) ${ }^{22} \mathrm{Na}^{+}$uptake was measured in oocytes injected with $\mathrm{H}_{2} \mathrm{O}$, wt (open bars) or mutant NCC cRNA (black bars). For all groups, transport assays were also performed in the presence of NCC blocker metolazone. Uptake values were shown as thiazide-sensitive $\mathrm{Na}^{+}$transport (wt was set as 100\%). 121: Glu121Asp; 392: Thr392lle; 442: Asn442Ser; 475: Ser475Cys; 489: Tyr489His; 751: Pro751Leu; 1030: Gln1030Arg; NCC glyc: glycosylated NCC; $\mathrm{kDa}$ : kilodalton. Data are presented as means \pm SEM. ${ }^{*} P<0.05$ indicates significant difference in relation to Wt NCC-injected oocytes.

cell membrane. To assess which of these processes are involved for each specific NCC mutant, the cell surface localization was examined.

\section{Surface expression of wild-type and mutant NCC}

$X$. laevis oocytes were injected with $\mathrm{H}_{2} \mathrm{O}$, and wild-type or mutant eGFP-NCC to study cell surface expression using confocal microscopy. ${ }^{27}$ Figure 3a depicts representative images of $X$. laevis oocytes injected with $\mathrm{H}_{2} \mathrm{O}$, and wild-type or mutant eGFP-NCC as stated, while Figure $3 \mathrm{~b}$ presents the combined densitometric quantification of three independent experiments ( $\sim 10$ oocytes/experiment), in which the total fluorescence of wild-type eGFP-NCC was quantified and set at $100 \%$. The total fluorescence of oocytes expressing wild-type eGFP-NCC proteins was significantly different from $\mathrm{H}_{2} \mathrm{O}$ injected oocytes ( $100 \pm 9 \%, n=44$ versus $6 \pm 1 \%, n=62$, respectively; Figure $3 \mathrm{~b}$ ). In addition, the surface localization of NCC was significantly impaired for some of the mutants, namely Thr392Ile (38 $\pm 5 \%$ ), Asn442Ser $(44 \pm 5 \%)$ and Gln1030Arg $(68 \pm 7 \%) \quad(n=26-53$; Figure $3 \mathrm{~b})$. The plasma membrane localization of the NCC mutants Glu121Asp $\quad(104 \pm 13 \%), \quad$ Ser475Cys $\quad(108 \pm 15 \%), \quad$ Tyr489His $(97 \pm 10 \%)$ and Pro751Leu $(110 \pm 16 \%)$ was not affected $(n=22-44$; Figure $3 \mathrm{~b}$ ), which points toward an intrinsic defect in NCC function as the reason for the observed decreased transport activity.

\section{DISCUSSION}

GS is an autosomal recessive salt wasting disorder characterized by hypokalemic metabolic alkalosis, and secondary hypomagnesemia and hypocalciuria (OMIM 263800). The underlying cause for GS is loss-of-function mutations and deletions in the SLC12A3 gene..$^{23,24,28}$
In the present study, the DNA of 163 unrelated patients with a clinical suspicion of GS were selected to screen for mutation by sequence analysis of the complete SLC12A3 coding and flanking regions. In patients with a single heterozygous mutation and in patients with a homozygous mutation, we subsequently analyzed all exons for the occurrence of deletions using MLPA.

Altogether, the GS phenotype was explained for 39 homozygous $(24 \%)$ and 99 compound heterozygous $(61 \%)$ patients. However, in 25 patients $(15 \%)$, a mutation in only one of the alleles was identified (Supplementary Table 1). The pattern of inheritance for GS is autosomal recessive, which is confirmed by the fact that heterozygous relatives are clinically and metabolically asymptomatic. For that reason, it is likely that there was a failure to detect a mutation on the other allele in case of the heterozygous patients. Genetic alterations can be located in gene regions that were not analyzed, such as promoter sequence, $3^{\prime}$ or $5^{\prime}$ UTR, or intronic sequences.

It has been described that patients with mutations in the CLCNKB gene, associated with Bartter syndrome, can present with a Gitelmanlike phenotype. In a preliminary study, 77 patients with no mutations in the SLC12A3 gene were tested for mutations in the CLCNKB gene (data not shown). In only five cases pathogenic mutations in the $C L C N K B$ gene have been identified, indicating that our failure to identify SLC12A3 mutations in this cohort of patients is probably due to misdiagnosis of the patients, and/or that these patients were sent in for exclusion of the diagnosis.

The SLC12A3 gene encodes the thiazide-sensitive NCC protein, which localizes to the luminal membrane of the DCT in the kidney. In these epithelial cells, NCC mediates the reabsorption of $\mathrm{Na}^{+}$and $\mathrm{Cl}^{-}$from the pro-urine to the intracellular compartment. As previously shown for $\mathrm{Na}^{+}$transporters such as NKCC2 and NCC, there are at least five potential mechanisms (class 1-5) by which mutations can reduce or abolish the activity of the transporter. ${ }^{16}$

Class 1 mutants exhibit decreased protein stability leading to a complete absence of the transporter due to improperly processed or unstable mRNA. This often results from promoter alterations, splice site mutations, premature stop codons or deletions. In the present study, we identified nine splice site mutations, four nonsense mutations and seven deletions (Supplementary Table 1; Table 1), which may result in abnormal splicing of the mRNA transcript, insertion of a premature termination codon or frame shift in the transcript, leading to a truncated mutant mRNA transcript, and therefore low levels of NCC protein expression. ${ }^{29}$ The effect of the c.(?_-6)_852+? and c.-66_23del deletions are unclear, as the start codon is within the deletion. For the c.-66_23del deletion it can be expected that either no protein is produced or that the translation initiation site has been moved downstream to Met53. The deletion of exons 1-6 (c.(?_-6)_852+?) probably disrupts protein production. The four intragenic deletions (exons 4-6, 5-6, 18 and 24) and the nine identified splice site mutations are predicted to lead to a frameshift and premature stopcodon. Owing to this premature termination (which also holds true for the four newly identified nonsense mutations), nonsense-mediated decay of the RNA is predicted and therefore probably no protein will be produced on these mutant alleles. The terminal deletion of exon 26 does not lead to nonsense-mediated decay, but probably will mimic missense mutations in the C-terminal part of the protein.

Additionally, we discovered 18 novel SLC12A3 missense mutations, which lead to amino-acid substitutions with an unknown effect on NCC function. We only included these mutations as pathogenic in case in silico analyses, together with other corroborating evidence, indicated that they could be classified as UV3 (probably pathogenic) or UV4 (pathogenic) according to the guidelines of the British and 
a

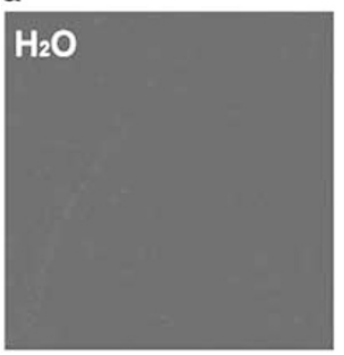

392

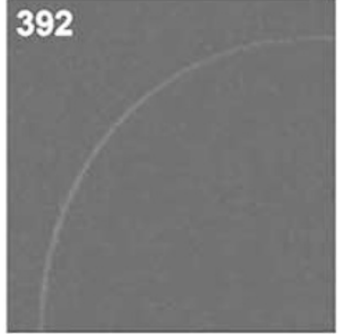

489

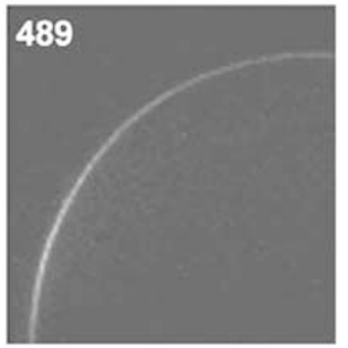

b

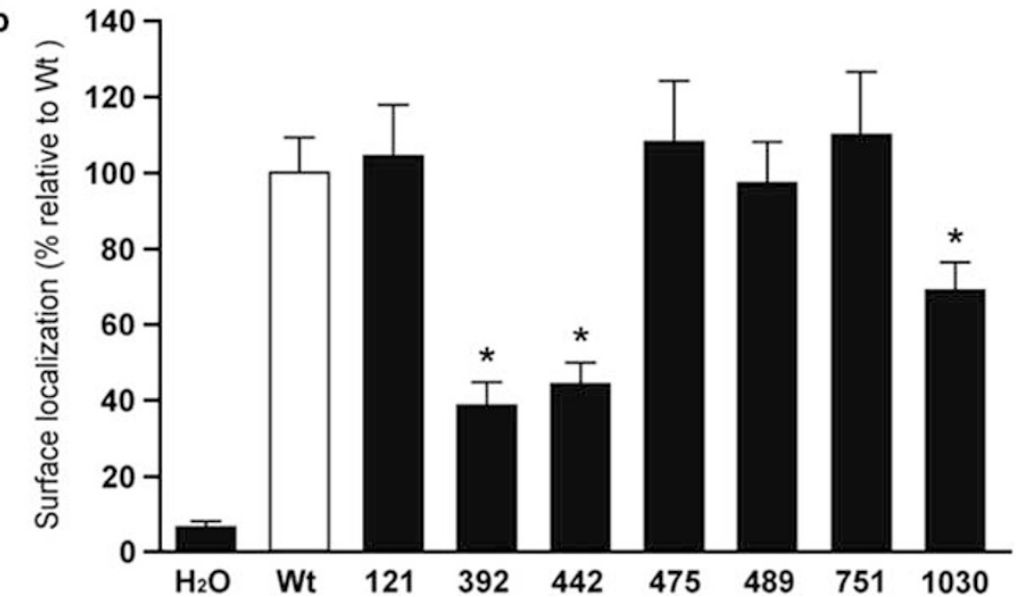

Figure 3 Surface expression of wt or mutant NCC protein in X. laevis oocytes, assessed by measuring fluorescence using laser-scanning confocal microscopy. (a) Representative confocal images of $X$. laevis oocytes expressing wild-type (wt) or mutant eGFP-NCC. (b) All wt (open bar) and mutant (black bars) images were quantified by measuring pixel intensity, while wt was set to 100\%. 121: Glu121Asp; 392: Thr392lle; 442: Asn442Ser; 475: Ser475Cys; 489: Tyr489His; 751: Pro751Leu; 1030: Gln1030Arg. Data are presented as means \pm SEM. ${ }^{*} P<0.05$ indicates significant difference in relation to wt NCC-injected oocytes.

Dutch clinical molecular biology societies. In all, five novel missense mutations and two previously described missense mutations ${ }^{30}$ were selected and introduced into NCC to study their effect on protein stability, cellular localization and uptake activity by use of the X. laevis oocyte as an established heterologous expression system (Figure 1). None of the mutations that were functionally characterized and presented in our manuscript have been identified in healthy controls or in SNP databases. In addition, the Glu121Asp, Thr392Ile and Asn442Ser mutations have been detected at least twice in non-related individuals in our patient group with clinical suspicion of GS.
Mutations in class 2 do not affect protein translation, but impair protein processing. As a result of misfolding, the NCC protein is retained in the ER and consequently targeted for degradation. Several studies have shown examples of class 2 NCC mutants. ${ }^{12-14}$ In our study, we identified one mutant with class 2 NCC mutant characteristics. The Thr392Ile mutation displayed severely reduced $\mathrm{Na}^{+}$uptake, which was identical to background (data not shown). Furthermore, the amount of Thr392Ile NCC mutant on the plasma membrane was severely decreased. Previously, Hoover et al ${ }^{27}$ showed that proper $\mathrm{N}$-linked glycosylation of both residues (N404 and N424) in NCC is 
Table 2 Summary of the different classes of NCC mutant in the present GS patients

\begin{tabular}{lcrc}
\hline & & Membrane & Mutant \\
& localization (\%) & class \\
\hline $\mathrm{H}_{2} \mathrm{O}$ & - & $6 \pm 1(n=44)$ & - \\
Wild-type & $100 \pm 8(n=65)$ & $100 \pm 9(n=62)$ & - \\
Glu121Asp & $27 \pm 3^{\#}(n=62)$ & $104 \pm 13(n=33)$ & 4 \\
Thr392Ile & $15 \pm 2^{\#}(n=53)$ & $38 \pm 5^{\#}(n=26)$ & 2 \\
Asn442Ser & $68 \pm 9^{\#}(n=64)$ & $44 \pm 5^{\#}(n=31)$ & 3 \\
Ser475Cys & $40 \pm 5^{\#}(n=75)$ & $108 \pm 15(n=26)$ & 4 \\
Tyr489His & $48 \pm 5^{\#}(n=58)$ & $97 \pm 10(n=26)$ & 4 \\
Pro751Leu & $54 \pm 4^{\#}(n=59)$ & $110 \pm 16(n=22)$ & 4 \\
Gln1030Arg & $58 \pm 6^{\#}(n=69)$ & $69 \pm 7^{\#}(n=53)$ & 3 \\
\hline Abbris & & & 4 \\
\hline
\end{tabular}

Abbreviations: GS, Gitelman syndrome; NCC, NaCl cotransporter.

The NCC mutant class (1-5) was determined by measuring NA+ uptake capacity (Figure 2 ) and cell surface localization (Figure 3 ) in Xenopus laevis oocytes, expressing wild-type or one of the seven selected NCC mutants (Table 1). Class (1) decreased protein stability; class (2) impaired protein processing of the cotransporter at the cell membrane; and class (5) accelerated protein removal or degradation.

\#indicates significant difference $(P<0.05)$ in relation to wild-type NCC-injected oocytes.

required for sufficient processing. The Thr392Ile mutation is situated in close proximity of these sites and may therefore hamper glycosylation. Interestingly, the upper band, which represents glycosylated NCC, seems to be (completely) absent for the Thr392Ile mutation on the western blot (Figure 1b). So, impaired glycosylation of the Thr392Ile mutant likely obstructs processing to the plasma membrane, and consequently diminishes the NCC transport activity.

Class 3 mutants disrupt insertion of an otherwise functional protein into the plasma membrane. These mutations are characterized by a reduced $\mathrm{Na}^{+}$uptake, and cytoplasm/ER and plasma membrane localization. Most previously identified NCC mutants belong to class $3^{12-14}$ and examples in our study are Asn442Ser and Gln1030Arg (Table 2). Presumably, these mutations produce NCC proteins of which the plasma membrane trafficking or internalization is affected. Interestingly, the Gln1030Arg mutation changes the last amino acid of NCC. Possibly, the Gln1030 amino acid is part of a protein-protein interaction motif, which is essential for trafficking.

Most of the mutations in our study belong to class 4 . The Glu121Asp, Ser475Cys, Tyr489His and Pro751Leu mutations did reach the plasma membrane; however, their $\mathrm{Na}^{+}$uptake rate was significantly reduced in comparison with wild-type NCC (Table 2). Notably, mutants Glu121Asp and Pro751Leu are situated within the $\mathrm{N}$ - and C-terminus of the protein (Figure 1b), suggesting a role in activation or regulation. The Ser475Cys and Tyr489His mutants are positioned in or nearby the large extracellular loop, which may indicate a role for these amino acids in ion affinity. None of the studied mutants meet the criteria of a class 5 mutation in which protein removal or degradation is accelerated.

Interestingly, substances that assist protein folding (chemical chaperones) have been successfully used to restore the trafficking and function of mutant proteins in case of the vasopressin type 2 receptor and cystic fibrosis transmembrane conductance regulator. ${ }^{31,32}$ It would be interesting to investigate chaperone-induced trafficking of mutant NCC proteins (Table 2), to ultimately use these chaperones as potential therapeutic agents. Altogether, this data contribute to the further understanding of the function and regulation of NCC. Future studies are needed to evaluate phenotype-genotype correlations to ultimately be able to predict, prevent or treat GS.

\section{CONFLICT OF INTEREST}

The authors declare no conflict of interest.

\section{ACKNOWLEDGEMENTS}

We are grateful to our colleague $\mathrm{H}$ Dimke for technical assistance and helpful suggestions. We thank all the patients, their families and the referring doctors for their participation. This study was financially supported by the Netherlands Organization for Scientific Research (ZonMw 9120.6110; ALW 700.55.302), a European Young Investigator award from the European Science Foundation (JGH), the Dutch Kidney foundation (C03.6017) (RJB), (C08.2252) (PS), The Swiss National Centre of Competence in Research (NCCR) Kidney Control of Homeostasis (Kidney.CH 507236) and the European Union Seventh Framework Programme (FP7/2007-2013; no 246539) (BG) and the European Community, FP7 (EUNEFRON 201590) (NVAMK).

1 Gitelman HJ, Graham JB, Welt LG: A new familial disorder characterized by hypokalemia and hypomagnesemia. Trans AssocAm Phys 1966; 79: 221-235.

2 Melander O, Orho-Melander M, Bengtsson K et al: Genetic variants of thiazide-sensitive $\mathrm{NaCl}$-cotransporter in Gitelman's syndrome and primary hypertension. Hypertension 2000; 36: 389-394.

3 Cruz DN, Shaer AJ, Bia MJ, Lifton RP, Simon DB: Gitelman's syndrome revisited: an evaluation of symptoms and health-related quality of life. Kidney Int 2001; 59: 710-717.

4 Spencer RW, Voyce MA: Familial hypokalaemia and hypomagnesaemia. A further family. Acta Paediatr Scand 1976; 65: 505-507.

5 Calo L, Punzi L, Semplicini A: Hypomagnesemia and chondrocalcinosis in Bartter's and Gitelman's syndrome: review of the pathogenetic mechanisms. Am J Nephrol 2000; 20: 347-350

6 Hisakawa N, Yasuoka N, Itoh H et al: A case of Gitelman's syndrome with chondrocalcinosis. Endocr J 1998; 45: 261-267.

7 Simon DB, Nelson-Williams C, Bia MJ et al: Gitelman's variant of Bartter's syndrome, inherited hypokalaemic alkalosis, is caused by mutations in the thiazide-sensitive Na-Cl cotransporter. Nat Gen 1996; 12: 24-30.

8 Gamba G, Saltzberg SN, Lombardi M et al: Primary structure and functional expression of a cDNA encoding the thiazide-sensitive, electroneutral sodium-chloride cotransporter. Proc Natl Acad Sci USA 1993; 90: 2749-2753.

9 Plotkin MD, Kaplan MR, Verlander JW et al: Localization of the thiazide sensitive $\mathrm{Na}-\mathrm{Cl}$ cotransporter, rTSC1 in the rat kidney. Kidney Int 1996; 50: 174-183.

10 Chobanian AV, Bakris GL, Black HR et al: The Seventh Report of the Joint National Committee on Prevention, Detection, Evaluation, and Treatment of High Blood Pressure: the JNC 7 report. JAMA 2003; 289: 2560-2572.

11 Ellison DH, Velazquez H, Wright FS: Thiazide-sensitive sodium chloride cotransport in early distal tubule. Am J Physiol 1987; 253: F546-F554.

12 De Jong JC, Van Der Vliet WA, Van Den Heuvel LP, Willems PH, Knoers NV, Bindels RJ: Functional expression of mutations in the human $\mathrm{NaCl}$ cotransporter: evidence for impaired routing mechanisms in Gitelman's syndrome. J Am Soc Nephrol 2002; 13: 1442-1448.

13 Kunchaparty S, Palcso M, Berkman J et al: Defective processing and expression of thiazide-sensitive $\mathrm{Na}-\mathrm{Cl}$ cotransporter as a cause of Gitelman's syndrome. Am J Physiol 1999; 277: F643-F649.

14 Riveira-Munoz E, Chang Q, Bindels RJ, Devuyst O: Gitelman's syndrome: towards genotype-phenotype correlations? Pediatr Nephrol 2007; 22: 326-332.

15 Sabath E, Meade P, Berkman J et al: Pathophysiology of functional mutations of the thiazide-sensitive $\mathrm{Na}-\mathrm{Cl}$ cotransporter in Gitelman disease. Am J Physiol Renal Physiol 2004; 287: F195-F203.

16 Gamba G: Molecular physiology and pathophysiology of electroneutral cation-chloride cotransporters. Physiol Rev 2005; 85: 423-493.

17 Koolen DA, Nillesen WM, Versteeg $\mathrm{MH}$ et al: Screening for subtelomeric rearrangements in 210 patients with unexplained mental retardation using multiplex ligation dependent probe amplification (MLPA). J Med Genet 2004; 41: 892-899.

18 Gamba G, Miyanoshita A, Lombardi M et al: Molecular cloning, primary structure, and characterization of two members of the mammalian electroneutral sodium-(potassium)chloride cotransporter family expressed in kidney. J Biol Chem 1994; 269: 17713-17722.

19 Cuppen E, van Ham M, Wansink DG, de Leeuw A, Wieringa B, Hendriks W: The zyxinrelated protein TRIP6 interacts with PDZ motifs in the adaptor protein RIL and the protein tyrosine phosphatase PTP-BL. Eur J Cell Biol 2000; 79: 283-293.

20 Moreno E, Cristobal PS, Rivera M, Vazquez N, Bobadilla NA, Gamba G: Affinitydefining domains in the $\mathrm{Na}-\mathrm{Cl}$ cotransporter: a different location for $\mathrm{Cl}$ - and thiazide binding. J Biol Chem 2006; 281: 17266-17275.

21 Pacheco-Alvarez D, Cristobal PS, Meade $\mathrm{P}$ et al: The $\mathrm{Na}+\mathrm{Cl}-$ cotransporter is activated and phosphorylated at the amino-terminal domain upon intracellular chloride depletion. J Biol Chem 2006; 281: 28755-28763. 
22 Syren ML, Tedeschi S, Cesareo $\mathrm{L}$ et al: Identification of fifteen novel mutations in the SLC12A3 gene encoding the $\mathrm{Na}-\mathrm{Cl}$ Co-transporter in Italian patients with Gitelman syndrome. Hum Mutat 2002; 20: 78.

23 Mastroianni N, Bettinelli A, Bianchetti $\mathrm{M}$ et al: Novel molecular variants of the $\mathrm{Na}-\mathrm{Cl}$ cotransporter gene are responsible for Gitelman syndrome. Am J Hum Genet 1996; 59: 1019-1026.

24 Lemmink HH, Knoers NV, Karolyi L et al: Novel mutations in the thiazidesensitive $\mathrm{NaCl}$ cotransporter gene in patients with Gitelman syndrome with predominant localization to the C-terminal domain. Kidney Int 1998; 54: 720-730.

25 Shao L, Liu L, Miao Z et al: A novel SLC12A3 splicing mutation skipping of two exons and preliminary screening for alternative splice variants in human kidney. Am J Nephrol 2008; 28: 900-907.

26 Vargas-Poussou R, Dahan K, Kahila D et al: Spectrum of mutations in Gitelman syndrome. J Am Soc Nephrol 2011; 22: 693-703.
27 Hoover RS, Poch E, Monroy A et al: N-glycosylation at two sites critically alters thiazide binding and activity of the rat thiazide-sensitive $\mathrm{Na}(+): \mathrm{Cl}(-)$ cotransporter. J Am Soc Nephrol 2003; 14: 271-282.

28 Simon DB, Lifton RP: The molecular basis of inherited hypokalemic alkalosis: Bartter's and Gitelman's syndromes. Am J Physiol 1996; 271: F961-F966.

29 Frischmeyer PA, Dietz HC: Nonsense-mediated mRNA decay in health and disease. Hum Mol Genet 1999; 8: 1893-1900.

30 Colussi G, Bettinelli A, Tedeschi S et al: A thiazide test for the diagnosis of renal tubular hypokalemic disorders. Clin J Am Soc Nephrol 2007; 2: 454-460.

31 Brown CR, Hong-Brown LQ, Biwersi J, Verkman AS, Welch WJ: Chemical chaperones correct the mutant phenotype of the delta F508 cystic fibrosis transmembrane conductance regulator protein. Cell Stress Chaperones 1996; 1: 117-125.

32 Robben JH, Sze M, Knoers NV, Deen PM: Functional rescue of vasopressin V2 receptor mutants in MDCK cells by pharmacochaperones: relevance to therapy of nephrogenic diabetes insipidus. Am J Physiol Renal Physiol 2007; 292: F253-F260.

Supplementary Information accompanies the paper on European Journal of Human Genetics website (http://www.nature.com/ejhg) 